Int. J. Electrochem. Sci., 16 (2021) Article ID: 210616

International Journal of

ELECTROCHEMICAL

SCIENCE

www.electrochemsci.org

\title{
CoO NPs/c-CNTs nanocomposite as electrochemical sensor for sensitive and selective determination of the carbofuran pesticide in fruits and vegetables
}

\author{
YanXun Yang ${ }^{1, *}$, You Zhou ${ }^{2}$, Yexing Liang ${ }^{3}$, Rui $W u^{4}$ \\ ${ }^{1}$ Biology section of Chongqing Bishan Laifeng middle school, Chongqing 402763, China \\ ${ }^{2}$ College of Biology and Food Engineering, Chongqing Three Gorges University, Chongqing 404120, \\ China \\ ${ }^{3}$ Agro-product Processing Institute, Chongqing Academy of Agricultural Science, Chongqing 401329, \\ China \\ ${ }^{4}$ Public resource trading center of Chongqin Bishan, Chongqing 402760, China \\ *E-mail: coriander_yang@ sina.com
}

doi: $10.20964 / 2021.06 .26$

Received: 24 January 2021 / Accepted: 22 March 2021 / Published: 30 April 2021

\begin{abstract}
This study was carried out for preparation and characterization of nanocomposite of CoO nanoparticles and carboxylated CNTs (CoO@c-CNTs NC) as electrochemical sensor for determination of the carbofuran pesticide in fruits and vegetables. The CoO@c-CNTs NC was prepared by dropping the centrifuged suspension of c-CNTs and $\mathrm{CoO}$ nanoparticles (CoO NPs) on activated glassy carbon electrode (GCE) as substrate and drying at room temperature. Morphological and structural studies of prepared NC using SEM and XRD exhibited the anchoring of rock-salt cubic phase of CoO NPs on cCNTs sheets which provided an effective electrical network for stabilization of well-distributed metal oxide NPs and higher effective surface area. Results of electrochemical studies with CV and DPV measurements showed that the high conductivity and large effective surface area of CNTs led to the higher electron transfer rate and higher current electro-oxidation of carbofuran in CoO@c-CNTs $\mathrm{NC} / \mathrm{GCE}$ than that of $\mathrm{CoO} \mathrm{NPs} / \mathrm{GCE}$. The linear range, detection limit and sensitivity values were obtained of $0-260 \mu \mathrm{M}, 0.004 \mu \mathrm{M}$ and $0.07275 \mu \mathrm{A} / \mu \mathrm{M}$ for detection of carbofuran, respectively. The comparison of obtained results for prepared carbofuran sensors with the other reported sensors showed that the detection limit and linear range of the CoO@c-CNTs NC/GCE were comparable or better than that of values obtained in some of reported carbofuran sensors. The study of interference effects of CoO@c-CNTs NC/GCE showed the good selectivity and anti-interference ability of the sensor for determination of carbofuran. The practical application of sensor for determination of the carbofuran pesticide in cabbages and oranges samples revealed that the carbofuran contents in the cabbages and oranges samples were estimated $0.66 \mu \mathrm{M}$ and $0.18 \mu \mathrm{M}$, respectively. Moreover, the obtained recovery and RSD values by the standard addition method indicated that the sensor had good accuracy for carbofuran detection in fruits and vegetables.
\end{abstract}


Keywords: Electrochemical sensor; Nanocomposite; CoO nanoparticles; Carboxylated CNTs; Pesticide; Carbofuran; Fruits; Vegetables

\section{$\underline{\text { FULL TEXT }}$}

(C) 2021 The Authors. Published by ESG (www.electrochemsci.org). This article is an open access article distributed under the terms and conditions of the Creative Commons Attribution license (http://creativecommons.org/licenses/by/4.0/). 